\title{
Recensement, description et examen critique des méthodes d'essais de cavitation sur modèle réduit
}

\section{Review, description and critical examination of cavitation test methods on scale models}

\author{
A report of Cavitation Working Group No. 1 of Société Hydrotechnique de France \\ PAR J. CHEVALTER, \\ E.D.F., STATION D'ESSAIS DE TURBINes,
}

\begin{abstract}
Ce texte a été mis au point dans le cadre de la Commission de la Cavitation de la Societe Hydrotechnique de France par le Groupe de travail $n^{\circ} 1$, dont la composition etait la suivante : MM. Chevalier, E.D.F., Station d'essais de turbines, Campmas, E.D.F., Division technique générale, Cazexave, Compagnie Nationale du Rhône, Fùras, Société des Forges et Ateliers du Creusot, et Ortune, Ateliers de Construction de Vevey.
\end{abstract}

\begin{abstract}
L'enquête conduite par un Groupe de travail de la Soctété Hydrolechnique de France a eu pour but de connaitre l'état actuel des moyens et des méthodes mis en cumre dans les laboratoires en ce qui concerne les essais de cavitation sar modèle rédnit. La première partie décrit les installations et les moyens d'essais en s'atturdant plus spécialement sur les problèmes de mesure de la teneur en air. La seconde partie fait une large part aux problèmes d'échelle et de simili. tude de cavitation. La comparaison des buts ponrsuivis par les laboratoires, selon qu'tls travaillent sur des turbines, des pompes ou des hélices, explique clairement la diversité des procédés utilisés en les justifiant. La troisième partie enfin traite de la présentation des résultats et analyse les méthodes actuelles de détermination du sigma critique. Devant leur diversité, une méthode graphique est proposée qui a l'avantage de permettre des comparaisons entre turbines et entre laboratoires et d'être appticable sans modification des installations existantes. La transposition au sigma d'installation, de mêtme que la part d'appréciation personnelle de chague constructeur, restent préservées par l'adjonction d'un terme correctif qui n'a pas à intervenir pour la comparaison au laboratoire.
\end{abstract}

Alors que la mesure des performances des turbomachines sur modèle réduit est à peu près semblable dans tous les laboratoires, aux différences d'appareillage et d'installation près, et que les méthodes de mesure en sont actuelle-

\begin{abstract}
The above working group has carried out a survey of present laboratory model cavitation research facilities and methods. The first part of this article gives a description of experimental means and inslallations, especially in respect of the measurements of amounts of air contained in the avaler. The second part discusses scale and cavitation similitude at length. A comparison between the respective aims of laboratories worling on turbines, pumps and propellers shows that their experimental procedures are both very varied and justified. The third part of the article diseusses the presentation of results and analyses present methods of determining critical sigma. In view of their diversity, o graphical method is suggested whereby comparisons between both turbines and laboratories can be made, and which does not require existing installations to be modified for its application: Transposition to the prototype sigma and the part left to the individual manufacturer's discretion are both preserved by applying a correction factor, which is not taken into account in the model comparison carried ont in the laborutory.
\end{abstract}

ment standardisées par la Commission Electrotechnique Internationale en ce qui concerne les turbines et les pompes, et par l'Internalional Towing Tank Conference en ce qui concerne les hélices, les méthodes d'essais des turbonachines 
à la cavitation semblent à l'heure actuelle bien plus dissemblables d'un laboratoire à l'autre, et jusqu'à présent, à notre connaissance, aucune tentative de standardisation les concernant n'a été entreprise du moins en ce (jui concerne les pompes et les turbines: le Code d'essais sur modèle réduit de la C.E.I. ne mentionnant le problème des essais de cavitation de turbines que pour mémoire.

C'est dans le but de préparer une standardisation des essais de cavitation des turbomachines que la Commission de la Cavitation de la Société Hydrotechnique de France a lancé une enquête auprès de différents laboratoires afin de connaitre leurs moyens, leurs méthodes et leur avis en ce qui concerne les essais de cavitation qu'ils effectuent.

Avec l'aide de ces documents, ce texte a été élaboré par un groupe de travail comprenant deux personnes travaillant en laboratoire, un exploitant de turbines industrielles, un maître d'ouvre et un constructeur.

Les organismes qui ont été contactés et qui ont bien voulu nous aider dans ce travail sont les suivants :

- Bassin d'essais des carènes, à Paris;

-- Electricité de France, Station d'essais de turbines;

- Etablissements Bergeron;

- Etablissements Chamilles;

-- Etablissements Escher Wyss;

_- Etablissements Franco Tosi;

- La SogréaH;

- Etablissements Vevey;

- Laboratoire d'Hydraulique de l'Ecole des Arts et Métiers, à Paris;

-. National Engineering Laboratory, à Glasgow.

\section{1. - LES INSTALLATIONS D'ESSAIS POUR L'ETUDE DES TURBOMACHINES A LA CAVITATION}

\section{A - Description du circuit d'essais.}

Le circuit d'essais est généralement un circuit fermé, c'est-à-dire sans passage de l'eau à l'atmosphère en aucun point de celui-ci; ce circuit fermé comporte toujours, en plus du modèle d'essais et des canalisations, une pompe permettant la circulation de l'eau à travers le modèle réduit de turbine ou d'hélice ou un organe absorbant la hauteur faite par le modèle réduit de pompe en essai.

Dans le cas des essais de turbines ou d'hélices, le fonctionnement en circuit fermé nécessite la présence d'un résorbeur, dans lequel les gaz dissous ou occlus libérés dans le modèle par la cavitation sont réabsorbés par l'eau au cours de son passage à faible vitesse dans une zone de haute pression. La plupart des installations en circuit fermé possèdent un résorbeur conçu en tant que tel ou simplement une longueur suffisante de canalisation de gros diamètre, à l'aval de la pompe de circulation, permettant un trajet relativement long du fluide dans une zone de forte pression.

Dans le cas d'essais de pompes, où la cavitation se produit à l'entrée de la roue, les bulles se résorbent automatiquement en passant dans la pompe d'essai et le résorbeur n'est plus nécessaire.

Les essais de cavitation de turbines peuvent être effectués sur un «circuit ouvert», e'està-dire avec passage de l'eau à l'atmosphère en un point du circuit. Ces installations correspondent à l'utilisation de roues de petit diamètre, et à de faibles débits. II est en effet alors possible de réaliser un circuit qui ne soit pas trop encombrant, malgré l'obligation de disposer d'un réservoir de décharge très haut, permettant la variation de la hauteur d'aspiration de la turbine par variation géométrique du plan d'eau dans ce réservoir.

En général, les installations en circuit ouvert ne possèdent pas de résorbeur, l'évacuation des gaz libérés devant s'effectuer par la surface libre au cours du passage du fluide à l'atmosphère. Cette surface libre est malheureusement souvent faible et il est nécessaire de s'assurer que l'on n'a pas recirculation de gaz en suspension.

$$
\text { **** }
$$

Dans le cas où il est intéressant de faire varier les conditions hydrauliques (chute ou vitesse d'écoulement) sur le modèle dans de grandes limites pour des coefficients de cavitation aussi variés que possible, un circuit du type fermé est mieux adapté qu'un circuit du type ouvert. En effet, le contrôle de la pression absolue audessus du modèle permet d'obtenir les coefficients de cavitation nécessaires quelles que soient les conditions d'écoulement réalisées. Un circuit fermé est d'autre part mieux adapté aux essais de modèle de grande dimension.

De toute façon, et quel que soit le type de circuit utilisé, il est nécessaire de ménager un contròle visuel de l'amenée d'eau au modèle, afin de s'assurer que la résorption ou l'évacuation des bulles est suffisante. 


\section{B - Méthodes de variation de la pression sta- tique.}

La variation de pression statique nécessaire à la variation du coefficient de cavitation est obtenue en diminuant la pression absolue audessus du modèle dans les installations en circuit fermé.

Cette variation de pression peut être obtenue de deux manières différentes:

- Soit fonetionnement continu des pompes à vide travaillant sur le circuit et réglage de la pression par une fuite ajustable à l'atmosphère (robinet ou soupape tarée);

- Soit branchement du circuit sur la pompe jusqu’à la bonne valeur de la pression, et ensuite isolement de celui-ci pendant la durée de l'essai, ce qui nécessite une chambre d'expérience particulièrement étanche.

Compte tenu des possibilités de vide maximal généralement limitées à des valeurs industrielles sur les installations de cavitation, on peut être amené à choisir les conditions d'écoulement (vitesse de l'eau ou chute d'essai) pour que le coefficient ou l'état de cavitation désiré puisse ètre atteint. Par exemple l'essai de cavitation sous faible chute d'une turbine dont le $\sigma$ critique est 0,5 , conduit à la réalisation d'une pression absolue à l'aval de $0,5 \mathrm{~m}$ d'eau (ce qui est un vide déjà poussé) si l'essai est réalisé sous $1 \mathrm{~m}$ de chute et seulement de $1,0 \mathrm{~m}$ d'eau, ce qui est bien plus facile à obtenir, si cet essai est réalisé sous $2 \mathrm{~m}$ de chute.

Signalons une possibilité d'essais qui pousse ce princípe à l'extrême et qui est quelquefois utilisée dans les essais de turbines où, à pression absolue sous la roue constante, on fait varier la chute pour régler le coefficient de cavitation.

En ce qui concerne les essais de turbines et selon la vitesse spécifique de la machine, la chute d'essais et la gamme des coefficients à explorer, il peut être nécessaire de mettre le modèle sous pression par rapport à l'atmosphère ou au contraire en dépression. Dans les installations en circuit fermé, permettant de réaliser des essais sous des chutes très différentes, compresseurs et pompes à vide sont ulilisés conjointement.

En effet, prenons l'exemple d'une roue de $n_{s} 300$ devant être essayée sous une chute de $50 \mathrm{~m}$ (chute industrielle). Si l'on veut explorer une zone s'étendant de quatre fois le a d'installation à 0,5 fois celui-ci, la pression absolue sur l'axe de la roue doit varier de $45 \mathrm{~m}$ à $5,5 \mathrm{~m}$ d'eau. Par contre, la même roue essayée sous une chute de $3 \mathrm{~m}$, dans la même gamme de $\sigma$, nécessite une variation de la pression absolue de $3 \mathrm{~m}$ à $0,3 \mathrm{~m}$.

Dans les installations d'essais de turbines en circuit ouvert, la hauteur d'aspiration est réglée par variation géométrique de la cote du plan d'eau dans le bassin de restitution du modele; une vanne-pointeau réglable et sensible sert alors à ajuster la perte de charge en fonction du débit et à régler le plan d'eau correspondant.

La variation de charge netle absolue à l'aspiration des pompes est quelquefois réalisce en agissant sur un étranglement dans la canalisation d'aspiration, de manière à en faire varier la perte de charge. Cette disposition est cependant a utiliser avec grande précaution, car l'écoulement à l'entrée de l'oillard peut en être tellement modifié que les conditions de cavitalion en soient altérées.

$$
* *
$$

Bien que la méthode utilisée dans les installations en circuit ouvert permettent un réglage plus stable et plus précis de la pression absolue dans la machine en essai, c'est l'utilisation de pompes à vide qui est le plus souvent adoptée pour sa facilité de mise en ouvre. Une précaution importante à prendre alors concerne l'implantation de la pompe de circulation, dans le cas d'un circuit d'essais de turbines on d'hélices. Il faut, en effet, éviter la cavitation sur la pompe, non seulement du point de vue de sa préservation mécanique, mais surloul eu égard aux fluctuations de régime qui pourraient être introduites dans le circuit par la cavitation qui $y$ prendrait naissance. Il ne faut pas perdre de vue également que, par ce procédé, les temps de passage du fluide dans les zones de basse pression sont considerablement augmentés par rapport aux temps de passage industriels, car la zone des faibles pressions est tres étendue et les vitesses d'écoulement sont faibles. De ce fait, l'influence de la teneur en air de l'eau du circuit peut être importante.

\section{C - Alimentation du modèle réduit.}

En ce qui concerne les pompes et les turbines, les mêmes impératifs que ceux précisés dans le Code d'essais de la Commission Electrotechnique Internationale sont à respecter.

Certains laboratoires d'essais d'hélices se préoccupent également de représenter sur le modèle une alimentation aussi semblable que possible aux conditions réelles. C'est ainsi que l'arbre de l'hélice peut être incliné sur l'horizontale jusqu’à des angles pouvant atteindre 15" et que l'hélice modèle peut être placée derrière une carène ou un générateur de sillage 
reproduisant le champ des vitesses dans lequel l'hélice travaille réellement.

$$
\text { 怗: }
$$

Les conditions d'alimentation jouent un rôle important dans l'apparition de la cavitation sur la machine essayée, puisque la modification de la répartition des angles d'attaque sur le profil en essai peut entrainer une modification des conditions de cavitation de celui-ci. C'est ainsi en particulier que, pour les turbines Kaplan, la plus grande altention doit être apportée au réglage de l'ouverture des pales of du distributeur, de maniòre à réaliser aussi exactement que possible la conjugaison qui a été déterminée au cours des essais de rendement. Pour les pompeshélices, les conditions d'alimentation dans la chambre d'eau sur le modèle sont à réaliser de manière aussi semblable que sur le prototype et l'éventuel effet d'une perte de charge réglable à l'aspiration du modèle (voir \$ B) doit être réduit au minimum.

\section{D-Propriétés de l'eau.}

L'eau doit être propre et claire et ne doit pas contenir de particules solides. Il est nécessaire d'éviter au maximum la présence de bulles d'air en suspension. (Code d'essais des turbines sur modèle réduit $\$$ 4.1.1.).

L'cflicacité du résorbeur, on de la portion de circuit permettant d'éviter le retour au modèle de turbine ou d'hélice des bulles s'étant formées lors d'un passage précédent, doit être contrôlée en cours d'essais.

L'appareillage nécessaire au contrôle de la teneur en air dissous, de l'eau du circuit, est actuellement prévu dans quelques laboratoires (voir \&E); il ne semble pourtant pas que l'on se préoccupe des moyens de la faire varier ou seulement de la maintenir constante. Si certains font leurs essais avec une eau désaérée au maximum, la plupart font l'essai sans précaution spéciale, c'est-à-dire avec de l'eau voisine de la saturation en air dissous; chaque point d'essai est alors effectué avec une teneur en air différente: celle qui correspond à la saturation de l'eau en air dissous sous la pression partielle correspondante.

En ce qui concerne les essais de pompes qui véhiculent normalement une eau aérée, les essais se font également en eau aérée. On doit alors prendre la précaution de travailler avec une charge absolue à l'aspiration suffisante pour que l'eau ne se dégaze que dans la zone de cavitation de la machine.

En fait, c'est l'existence de bulles gazeuses non dissoutes qui constitue les points faibles par lesquels la cavitation débute. Ces impuretés gazeuses, ou noyaux, ont une influence au moins égale à celle de la teneur en air dissous. L'équipement nécessaire à la mesure courante de cet air occlus est malheureusement encore inexistant dans les laboratoires. Cependant, afin d'éviter un dégazage de l'eau sous basse pression, qui risquerait de créer des bulles d'air ou des noyaux gazeux en suspension et masquerait le phénomène de cavitation, certains laboratoires opèrent avec une eau préalablement désaérée; c'est en particulier le cas des essais de pompes de condenseur qui véhiculent normalement une eau désaérée. Ce procédé est cependant à utiliser en général avec prudence, car pour les très faibles teneurs en gaz dissous, il est prouvé que la cavitation se produit difficilement. Dans quelques laboratoires, un léger apport permanent d'eau fraiche, compensé par une déperdition égale vers l'extérieur, permet justement d'éviter cet inconvénient.

$$
* *
$$

La température de l'eau du circuit est toujour's mesurée au voisinage du modẻle, de manière à connaitre avec précision la tension de vapeur correspondante (*); cependant en général aucune régulation de température n'est installée, seul un troncon de circuit particulièrement bien réfrigéré évacue les calories dissipées pendant les essais.

Rappelons à ce sujet que le Code d'essais des turbines sur modèle réduit limite la température maximale des essais à $35^{\circ}$, ce qui, après une période prolongée d'essais en circuit fermé, impose la présence d'une portion de circuit permettant un refroidissement de l'eau. Le dispositif que nous avons signalé précédemment, c'est-à-dire l'injection d'une quantité d'eau fraîche compensée par une évacuation égale, permet une régulation simple de la température en plus de son rôle concernant la teneur en air.

\section{E-Appareillage et méthodes de mesure par- ticuliers aux essais de cavitation.}

Les appareils et méthodes de mesure particuliers aux essais de cavitation sont assez semblables d'un laboratoire à l'autre, qu'il s'agisse des mesures de vide, de pression statique, de température de l'eau ou de pression barométrique; seule, la mesure de la teneur en air diffère sensiblement d'un laboratoire à l'autre.

(") Rappelons que la tension de vapeur n'est pas dans les pompes un terme toujours aussi faible que dans les tarbines et les hélices : pompes à eau chaude, pompes a hydrocarbures. 
La plupart des laboratoires se préoccupant de la question utilisent, à titre d'étalonnage d'un autre appareil ou de contrôle de l'état de l'eau du circuit, la méthode chimique dite de «Winkler ». Elle consiste à doser l'oxygène contenu dans l'eau d'un prélèvement, par une méthode iodométrique. Un hydroxyde de manganèse absorbe l'oxygène dissous dans l'eau du prélèvement pour se transformer en acide manganeux. La réaction de cet acide faible sur de l'acide sulfurique et de l'iodure de potassium permet la libération d'iode, qui sera dosé par les procédés habituels d'iodométrie. Cette nuéhode, relativement précise, a l'inconvénient d'être longue, de nécessiter des solutions titrées de conservation difficile et d'obliger à des prélèvements devant être effectués en général sous vide, sans réaération de l'eau prélevée.

On calcule ensuite la teneur en air dissous par des abaques faisant intervenir la température de l'eau et la pression à laquelle le prélèvement a été effectué.

Un autre procédé, employé dans quelques laboratoires, permet l'extraction de l'air par une séparation physique. Le prélèvement est introduit dans un appareil permettant de le faire cascader sous vide plusieurs fois sur des colonnes à plateau et de séparer le gaz de l'eau. Ce dispositif a l'avantage de permettre la mesure directe de la teneur en air et non plus de l'oxygène; il présente par contre l'inconvénient d'obliger à travailler sur quelques centimètres cubes de prélèvement.

Signalons enfin l'utilisation possible de certains doseurs d'oxygène dissous dans l'eau, utilisés dans les centrales thermiques. Ils permet- tent d'obtenir un enregistrement continu de la teneur en oxygène, mais sont à adapter à la gamme d'utilisation en laboratoire où l'eau est en général saturée, alors qu'elle est voisine de la désoxygénation complète dans la centrale thermique.

$$
\text { * }
$$

Ces procédés ne permettent que la mesure des gaz dissous, alors qu'il serait d'un intérêt plus grand de pouvoir caractériser la teneur en noyaux de cavitation dans l'eau utilisée. Signalons la possibilité de dénombrer ces novaux en mesurant, par exemple, l'absorption d'une énergie de fréquence ultrasonore à la traversée d'une capacité remplie d'eau (Strasberg). Les noyaux de rayon $R_{0}$ ont une fréquence propre:

$$
\begin{aligned}
f_{0} & =\frac{\sqrt{3 \gamma\left(\mathrm{P}_{0} / \rho\right)}}{2 \pi \mathrm{R}_{0}} \\
\mathrm{P}_{0} & =\text { Pression dans le noyau; } \\
\gamma & =\text { Rapport des chaleurs spécifiques du gaz; } \\
\vartheta & =\text { Masse spécifique. }
\end{aligned}
$$

Les ultrasons de fréquence $f$ voisine de $f_{0}$ meltent en résonance les noyaux de rayon voisin de $R_{0}$. L'absorption d'énergie dans la bande de fréquence $f_{0}$ est alors proportionnelle au nombre de noyaux de diamètre $R_{0}$.

Quoi qu'il en soit, il parait souhaitable que la teneur en air soit mesurée et consignée sur les feuilles de relevé pour chaque série d'essais, afin que les comparaisons entre deux installations puissent être effectuées pour des conditions connues de l'aération de l'eau du circuit.

\section{II. - LA SIMILITUDE DANS LES ESSAIS DE CAVITATION DE TURBOMACHINES}

\section{A. Choix du diamètre minimal de la machine d'essai.}

Le diamètre minimal possible doit satisfaire aux exigences pratiques d'une reproduction geométriquement semblable à la machine industrielle et d'une possibilité d'usinage soigné conduisant à l'identité entre elles des différentes aubes de la même roue.

routes les installations d'essais de turbine que nous avons étudiées tiennent compte des prescriptions qui sont notifiées dans le code d'essais des turbines sur modèle réduit, c'estàdire diametre minimal de roves : $250 \mathrm{~mm}$. Le choix du diamètre d'une hélice modòle est évidemment conditionné par des considérations d'usinage identiques à celles rốgissant les pom- pes et les turbines; cependant, la nécessité d'éliminer au maximum l'efret de la paroi du tunnel d'essai sur l'holice modele, ainsi que la nécessité de ne pas avoir de modèles de turbines trop importants, conduisent en général, pour des dimensions raisonnables d'installation, à des hélices de petit diamètre, par exemple 200 à $300 \mathrm{~mm}$. Des considérations semblables sur les dimensions de l'installation d'essais limitent le diametre des roues d'essais de pompes a 200 ou $300 \mathrm{mmn}$.

\section{B-Choix de la similitude à utiliser.}

Le choix de la similitude à utiliser pour les essais de cavitation de turbomachines a déjà fait l'objet de nombreuses publications et les diffé- 
rentes installations d'essais traduisent les différences de points de vue sur cette question. En plus des paramètres de similitude qui interviennent dans l'étude normale des performances, il s'introduit, quand il y a cavitation, un paramètre de similitude supplémentaire.

\section{Coefficient de Thoma.}

Le coefficient de Thoma est un nombre sans dimension qui permet de caractériser l'installation d'une machine hydraulique. Dans le cas d'une turbine, il exprime l'écart entre la charge aval et la tension de vapeur, rapporté à la chute.

$$
\sigma_{\mathrm{Thoma}}=\frac{\mathrm{H}_{a}-\mathrm{H}_{v}}{\mathrm{H}}
$$

$\mathrm{H}_{a}=$ Hauteur d'eau équivalente à la charge;

$\mathrm{H}_{2}=$ Hauteur représentative de la tension de vapeur;

$\mathrm{H}=$ Chute nette.

C'ẹst une forme particulière du coefficient de cavitation $\sigma$ :

$$
\sigma=\frac{p_{0}-p_{v}}{1 / 2 \varphi v^{2}}
$$

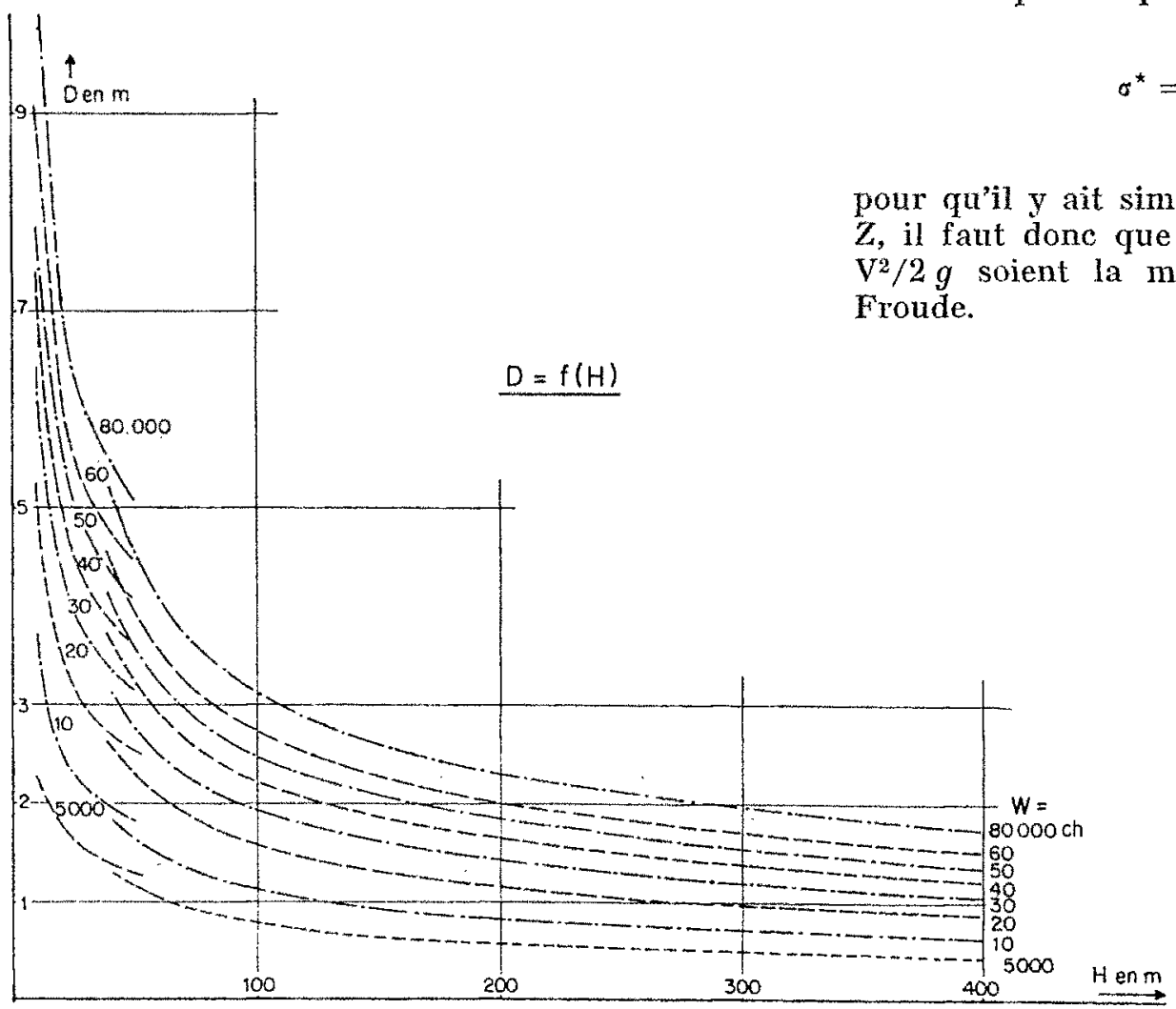

Fig. 1.

Diamètre de rouve

d'une turbine

en fonction de la chute

(Statistiques E.D.F.). exprimant l'écart entre la pression absolue et la tension de vapeur, rapporté à la dynamique de l'écoulement.

\section{Similitude de Froude.}

En un point $\mathrm{M}$ donné, la pression étoilée $p^{\star}$ est définie par :

$$
p^{\star}=p_{0}+\varepsilon g Z
$$

où :

$p_{0}$ est la pression statique;

et :

$Z$ la cote du point $M$ par rapport à la réfé-

C'est la pression étoilée $p^{\star}$ qui intervient dans les formules de similitude. Le coefficient de cavitation qui aura la même valeur sur le modèle et sur le prototype sera :

$$
\sigma^{\star}=\frac{p^{\star}-p_{v}}{1 / 2 V^{2}}
$$

il a done pour expression :

$$
\sigma^{*}=\sigma+\frac{2 g Z}{V^{2}}
$$

it similitude des o quelle que soit que l'échelle des $Z$ et celle des $V^{2} / 2 g$ soient la même. C'est la condition de Froude. 
Cette condition impose donc de représenter les gradients de pression statique et dynamique à la même échelle.

Elle lie les dimensions du modèle réduit (D) à la vitesse de l'écoulement (U), ou à la chute (H) d'essai pour une turbine. De ce fait, les dimensions des installations d'essais étant limitées, elle conduit à travailler avec de faibles vitesses, donc avec des nombres de Reynolds bas, en satisfaisant à la relation :

$$
\left(\mathrm{V}^{2} / \mathrm{D}\right)_{\text {modele }}=\left(\mathrm{V}^{2} / \mathrm{D}\right)_{\text {industriel }}
$$

La relation de Froude a cependant une infuence négligeable sur les phénomènes de cavitation pour des installations n'ayant pas de dimension verticale importante, ce qui permet d'augmenter la vitesse d'écoulement ou la chute, done le nombre de Reynolds.

\section{Pratiques n'essars pour les hélices, Les TURBINES ET LES POMPES.}

En ce qui concerne les inslallations d'essais d'hélices, le choix de la similitude est en général guidé par les principes suivants : les dimensions de l'installation devant rester limitées et l'eftet de paroi évité au maximum, on est conduit à une hélice de petit diamètre, pour laquelle le respect de la condition de Froude est saus influence. On peut donc alors augmenter la vitesse du courant sans satisfaire à la condition $\left(\mathrm{V}^{2} / \mathrm{D}\right)_{\text {modele }}=\left(\mathrm{V}^{2} / \mathrm{D}\right)_{\text {real }} ;$ donc augmenter le nombre de Reynolds et éviter un plan d'ean libre représentant la surface, qui devrait en général être maintenu à quelques dizaines de centimètres seulement au-dessus du modèle.

$$
\text { *3: }
$$

Dans les installations d'essais de turbines à la cavitation, la plus grande diversité est rencontrée dans la valeur usuelle du rapport $H / D$ (chute d'essai rapportée au diamètre de la roue) réalisable à la Station d'essais. Ce paramétre, que nous substituerons dans ce qui suit au nombre de Froude, a l'avantage de caractériser à la fois l'installation d'essais el la machine industrielle dans un domaine de puissance donnée (voir fig. 1).

- Un H/D faible caractérise une installation pouvant essayer des roues de grand diamètre $(500 \mathrm{~mm})$ sous des chutes d'essais faibles $(1 \mathrm{~m}): \mathrm{H} / \mathrm{D}=2$.

Il caractérise également une machine de forte vitesse spécifique $\left(n_{s}=700\right)$ et de puissance moyenne $(20000 \mathrm{ch})$, pour laquelle la chute industrielle est faible et le diamètre de roue élevé $(H=10 \mathrm{~m}, \mathrm{D}=5 \mathrm{~m}): \mathrm{H} / \mathrm{D}=2$.
- Un $H / D$ ćlevé caractérise une installation d'essais pourant essayer des rones de petit diamètre $(300 \mathrm{~mm})$ sous des chutes d'essais élevées $(50 \mathrm{~m})$; on a alors $\mathrm{H} / \mathrm{D}=150$.

II caractérise également une machine de vitesse spécifique faible $\left(n_{s}=90\right)$, de puissance moyenne $(30000 \mathrm{ch})$, pour laquelle la chute industrielle atteint $200 \mathrm{~m}$ et le diamètre $1,35 \mathrm{~m}: \mathrm{H} / \mathrm{D}=150$.

Les premières de ces installations permettent d'essayer des machines Kaplan de basse chute et grand diamètre au même nombre de Froude (mème $\mathrm{H} / \mathrm{D}$ ); les secondes ne se préoccupent pas de cette condition et adaptent, dans les limites de l'installation, le diamètre du modele $(300$ à $350 \mathrm{~mm}$ ) ou la hauteur de chute, de manière à réaliser l'essai dans les meilleures conditions permises par la plate-forme (puissance et débit).

Signalons que, parmi les premières de ces installations, la plupart sont équipées de maniere à fatre varier la chute d'essais dans d'assez grandes limites (de 1 à 10 par exemple), ce qui permet également d'essayer les turbines Kaplan à la cavilation sous la chute industrielle, c'està-dire avec des vilesses d'écoulement égales aux vitesses réelles.

Les secondes de ces installations pourmient essayer en similitude de Froude des machines de vitesse spécifique faible, pour lesquelles cette condition n'est plus a respecter, compte tenu des dimensions verticales réduites de la rouo vis-à-vis de la pression statique.

$$
\text { *t: }
$$

Les essais de pompe sont, eux, en général, efrectués en réalisant sur le modèle la même hanteur que sur la pompe réelle $V_{1}=V_{2}$, ce qui est un moyen terme entre la similitude de Reynolds $\mathrm{V}_{1} \mathrm{D}_{\mathrm{i}}=\mathrm{V}_{2} \mathrm{D}_{2}$, la similitude de Froude $V_{1} \sqrt{D_{2}}=V_{2} \sqrt{D_{1}}$ et la similitude des temps de passage $V_{1} \vec{D}_{2}=V_{2} D_{1}$. Cette pratique peut se justifier par les raisons suivantes :

- Les modèles de pompes sont en général plus pelits que les modeles de turbines, pour des questions de dimension des installations d'essais, et un nombre de Reynolds suffisant impose une hauteur élevée;

- Les dimensions verticales de la roue à l'entrée sont en général faibles, môme pour les machines a axe horizontal;

--.. Certaines formes de cavitation physique dans le liquide apparaissant aux faibles débits ne sont décelables que par le bruit en l'absence de visualisation; il faut donc un niveau de pression suffisant pour éviler que ce bruit ne tombe dans des zones inaudibles ot quo cette cavitation passe inapereue, alor's qu'elle réapparaîtrait sur la vraie machine. 
Alors que, pour les essais de rendement sur modèle réduit, la chute d'essais peut être choisie dans de larges limites, car son influence sur les résultats est trós réduite pourvu que le nombre de Reynolds soit suffisant, d'autres considérations doivent intervenir dans les essais de cavitation.

La représentation en similitude des pressions statiojues, c'est-à-dire le respect du paramètre de Froude, est, nous l'avons montré, la condition fondamentale d'une représentation correcte des figures de cavitation lorsque les dimensions verticales de linstallation sont grandes vis-a-vis des charges statiques absolues. C'est notamment le cas des essais de turbines Kaplan de grand diametre de roue et de faible chute et a fortiori des groupes à axe horizontal de faible chute (groupes bulbes). Dans ce cas, en effet, le diamètre de la roue peut représenter la moitié de la hauteur d'eau équivalente à la pression statique et la figure de cavitation est évidemment fonction de la bonne représentation des pressions statiques.

Dans tous les cas où l'essai aura pour but de localiser les figures de cavitation, en vue notamment. de déterminer les régions de la pale exposées au risque d'érosion, il sera nécessaire d'opérer avec un modele de diamètre assez grand, d'installer ce modèle avec la même position d'axe de rotation que la machine réelle et de respecter le nombre de Froude (la valeur du paramètre $H / D$ pour les turbines). Cette condition est également nécessaire lorsque l'essai doit déterminer l'évolution des caractéristiques énergetiques de la machine (poussée, rendement, puissance ...) en fonction de l'apparition et du développement des figures de cavitation.

Cette condition ne pourra etre abandonnée que lorsque les dimensions verticales de la machine deviennent faibles par rapport aux pressions statiques. Pour les turbines par exemple, il sera possible de ne pas opérer dans ces conditions lorsque la valeux du paramètre $H / D$ nominal industriel est supérieur à 4 , ce qui revient à admettre que, pour des machines Kaplan verticales, une variation de pression statique de part et d'autre de l'axe de rotation des pales inférieure à $10 \%$ n'influence pas la représentation de la figure de cavitation.

Le respect de la similitude des pressions absolues n'est pas impératif, d'autre part, si l'essaí de cavitation est conduit uniquement dans le but de contrôler ou de fixer l'implantation de la machine industrielle. C'est ce qui explique que les laboratoires qui essayent des hélices ne se préoccupent pas en général de la position du plan d'eau au-dessus de l'axe de l'hélice modèle, bien que les dimensions de celle-ci soient en général grandes par rapport à la pression absolue. En effet, dans ce cas, on arrête l'essai dès que la figure de cavitation recherchée apparaît et on ne s'intéresse pas en général à son développement sur l'hélice.

Le coefficient de cavitation ainsi déterminé sur le modèle sera comparé à sa valeur d'exploitation industrielle pour le point le plus haut de la roue réelle, l'inexactitude résultant de ce procédé introduisant ainsi une marge de sécurité dans le contrôle. Ceci suppose cependant que les differentes figures de cavitation sont independantes les unes des autres et ne permet pas d'étudier de facon étendue la variation des performances énergétiques de la machine en fonction du $\sigma$. Cette solution présente l'avantage de nécessiter des modeles de diametre plus faible, de travailler avec des vitesses d'écoulement plus élevées et de mesurer des puissances importantes.

\section{$* *$}

En résumé, si l'on veut éviter toute cavitation sur le rél et la déceler sur le modèle dès ses premiè'es manifestations, l'essai pourra être effectué sans respecter la similitude de Froude, même s'il s'agit d'une machine de dimension verticale importante. C'est le cas des pompes, dont on n'admet pas le fonctionmement en cavitation, car leurs caractéristiques sont rapidement perturbées par la cavitation qui se développe toujours à l'entrée de la roue, ou des hélices marines pour lesquelles le bruit est une manifestation gênante de la cavitation.

Si l'on veut, par contre, étudier sur le modèle le développenent de la cavilation tel qu'il se produira sur le réel, lout au moins tant que les Inis générales de la similitude restent valables, il est nécessaire, pour les machines de grande dimension verticale, d'effectuer l'essai dans des conditions voisines de la similitude de Froude.

Quelle que soit cependant la méthode utilisée, il semble nécessaire que le type de similitude adopté soit précisé dans le rapport d'essais (valeurs des pressions absolues et des vilesses d'écoulement réalisées). Dans tous les cas où cela sera possible, il est recommandé de réaliser quelques points de régime pour des valeurs de pression absolue et de vitesse d'écoulement aussi différents que possible, de manière à s'assurer que le donnaine où les essais ont été effectués n'est pas soumis à un effet d'échelle trop important.

\section{C - Disposition du modèle réduit.}

Dans le cas des essais de turbines ou de pompes, il n'est pas toujours possible d'essayer le modile réduit avec la mène direction d'axe de 
rotation que pour Ia machine industrielle. Un changement dans la direction de l'axe de rotation n'a en principe pas d'influence tant que le diamètre de la roue * soit réelle, soit modèle réduit - qui a été installée avec son axe horizontal, c'est-à-dire la différence de cote entre les points haut et bas de cette roue dans sa partie basse pression, reste faible devant la pression absolue qui règne à cet endroit.

$$
* * *
$$

Ces conditions sont intimement liées au problème de la similitude que nous venons d'étudier. Rappelons done qu'un essai de ponpe ou de turbine conduit sans respecter les conditions citées plus haut ne permettra pas de porter un jugement précis sur la localisation el l'étendue des figures de cavitation susceptibles d'apparaître sur la machine industrielle ainsi que sur les altérations de rendement qu'elles entrainent. Une telle disposition ne sera acceptable que lorsque l'essai de cavitation sera entrepris uniquement dans le but de contrôler ou de fixer l'implantation de la machine industrielle.

\section{D- Etats de surface.}

Les surfaces, jouant un rôle prépondérant dans le fonctionnement de la machine, doivent être telles que l'on puisse considérer qu'elles ont la meme rugosité que celles de la machine industrielle code d'essais international des turbines sur modẻle réduit).

Un grand soin doit être apporté dans la réalisation des raccordements entre pièces devant être réunies ensemble, de manière à éviter tout décollement local pouvant être to siege d'une amorce de cavitation particuliè au modele.

Les defauts locaux sur les pales ef notamment sur les bords d'attaque doivent être évités avec soin, de manic̀re à ne pas constituer les points fables par où la cavitation pourrait samorcer. Ceci est particulièrement nécessaire dans le cas ou on caractérise la cavitation par lapparition de figures particulières; il est alors nécessaire de s'assurer que des accidents locaux sur les pales, ou des impuretés se collant sur le bord d'attaque, n'influencent pas l'apparition du phénomène.

\section{III. - DETERMINATION DU SEUIL DE CAVITATION}

\section{A - Paramètre de cavitation.}

Quelle que soit linstallation, le processus d'essais est identique : le point de fonctionnement étant réglé et stabilisé. on diminue progressivement la pression statique par le procédé spécifique à chaque plate-forme et on relève les perfornuances du modèle ou son aspect en les reliant à la valeur du paramètre de cavitation correspondant. Nous avons défini au chapitre II le coefficient de cavitation sous sa forme la plus générale :

$$
\sigma=\frac{p_{0}-p_{v}}{\rho V^{2} / 2}
$$

dans lequel :

$p_{0}=$ Pression statique absolue au point oì $\sigma$ est calculé;

$p_{v}=$ Tension de vapeur;

$\mathrm{CV}^{2} / 2=$ Pression dynamique de référence correspondant à une vitesse d'écoulement caractéristique facile à déterminer.

$\sigma$ est calculé sous cette forme pour les études d'hélices de vannes ou de profils fixes.

Dans létude des turbines, il est utilisé sous une forme légèrement différente, celle de Thoma :

$$
\sigma=\frac{\mathbf{H}_{a}-\mathbf{H}_{s}-\mathbf{H}_{v}}{\mathbf{H}}
$$

$H_{a}=$ Hauteur représenlative de la pression almosphéricque;

$H_{s}=$ Hautenr d'aspiration complée positivement quand la roue est au-dessus du niveau aval:

$\mathrm{H}_{v}=$ Hautem représenlative de la lension de vapeur:

$\mathrm{H}=$ Chute nelte.

Pour certaines éludes relatives au fonctionnement des turbomachines, on ulilise d'ailleurs quelquefois le paramètre $\bar{z}$ sous sa forme (1) où $V$ est remplacé par la vitesse relative $W$.

En ce qui concerne les pompes el bien que le paramctre $\sigma$ soit également ulilisé, sa définition fait intervenir: un terme d'un usage courant dans l'étude des pompes, la charge nette absolue à J'aspiration (NPSH).

$$
(\mathrm{NPSH})=\mathrm{H}_{a}+\mathrm{H}_{3}-\mathrm{J}_{a}-\mathrm{H}_{\mathrm{V}}
$$

avec : 
${ }^{3} \mathrm{H}_{a}$ Hauteur de liquide pompé représentative de la pression totale (gaz + vapeur) régnant au-dessus de la surface libre à l'aspiration;

$\mathrm{H}_{s}$ Différence d'altitude entre la surface libre et le point où (NPSH) est calculé;

$J_{a}$ Perte de charge à l'aspiration entre le réservoir el la pompe, mesurée en hauteur de liquide traversant la tuyauterie;

$\mathrm{H}_{v}$ Hauteur représentative de la pression vapeur.

On a alors :

$$
\sigma=\frac{(\mathrm{NPSH})}{\mathrm{H}}
$$

Ce terme n'est cependant interessant que pour l'utilisateur qui ne connait que les conditions de fonctionnement globales de sa pompe. Précisons de plus qu'il doit être calculé en faisant intervenir la hauteur manométrique totale dès que la cavitation a altéré les performances, car l'altération de la hauteur faite par la pompe ne doit pas intervenir dans le calcul de $\sigma$.

C'est pourquoi les constructeurs utilisent plutòt le paramètre

$$
\omega_{a}=\frac{(\mathrm{NPSH})}{\left(\mathrm{V}_{a}^{2} / 2 g\right)}
$$

$V_{a}$ étant la vitesse d'entraînement au diamètre d'entrée de la roue.

Ce paramètre caractérise l'œillard de la roue indépendamment de sa sortie et du reste de la machine. Il pernet notamment de passer des essais de cavitation d'une roue aux caractéristiques d'une roue semblable et de rognage différent, car $H$ lié à la sortie de la roue n'y intervient plus.

\section{B - Présentation des résultats.}

La prósentation des résultats d'essais diffère selon le type de machine essayé et également selon le laboratoire d'essais; les courbes tracées traduisent cependant en général l'évolution d'un paramètre caractérisant je point de fonctionnement de la machine, en fonction d'un paramètre caractérisant l'état de cavitation.

\section{HÉliCes.}

La présentation la plus courante consiste à tracer les courbes du degré d'avance:

$$
\lambda=\frac{V}{n D}
$$

$V=$ Vitesse de l'eau;

$n=$ Vitesse de rotation;

$\mathrm{D}=$ Diamètre de l'hélice.

en fonction du paramètre o défini au $\$$ A pour lapparition de chaque type de cavitation (fig. 2).

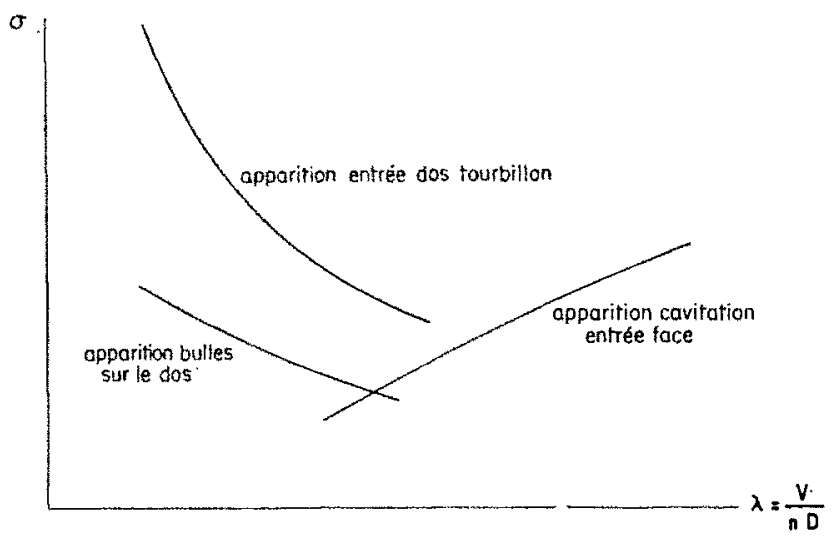

Fic. 2

Courbes typiques de cavitation d'une hélice.

Courbes d'apparition des diverses figures de cavitation.

Le coefficient $\sigma$ est calculé soit pour la pointe supérieure de l'hélice, soit pour l'axe de l'hélice.

Lorsqu'on étudie l'altération des performances, on trace l'évolution du rendement et des coeffcients de couple $C_{Q}$ et de poussée $C_{n}$ en fonction de $\lambda$. L'ensemble de ces résultats pour des vitesses, donc des $\sigma$, variables peut se représenter dans un diagramme tel que celui de la figure 3.

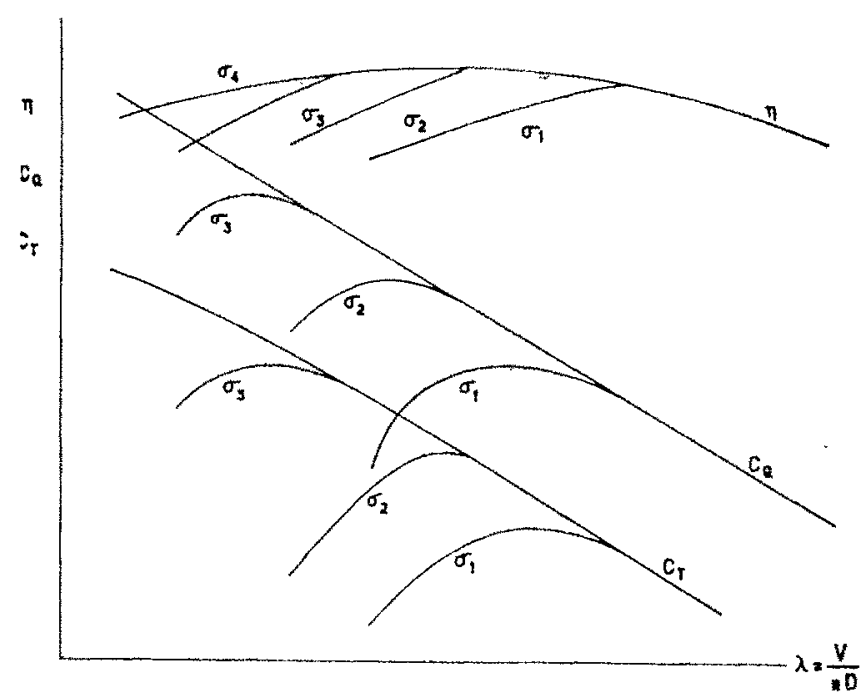

FIG. 3

Courbes typiques de cavitation d'une hélice Altération de l'ensemble des performances de l'hélice. 


\section{Turbines.}

On trace en général l'évolution d'un paramètre de puissance ou de débit et du rendement en fonction du paramètre o défini au $\S \mathrm{A}$ pour chacun des points de fonctionnement étudiés de la colline.

Le long de cette courbe, on note, au fur et à mesure de son évolution, l'apparition des diverses manifestations de cavitation (bruit, aspect, ...) (fig. 4); l'ensemble des résultats est

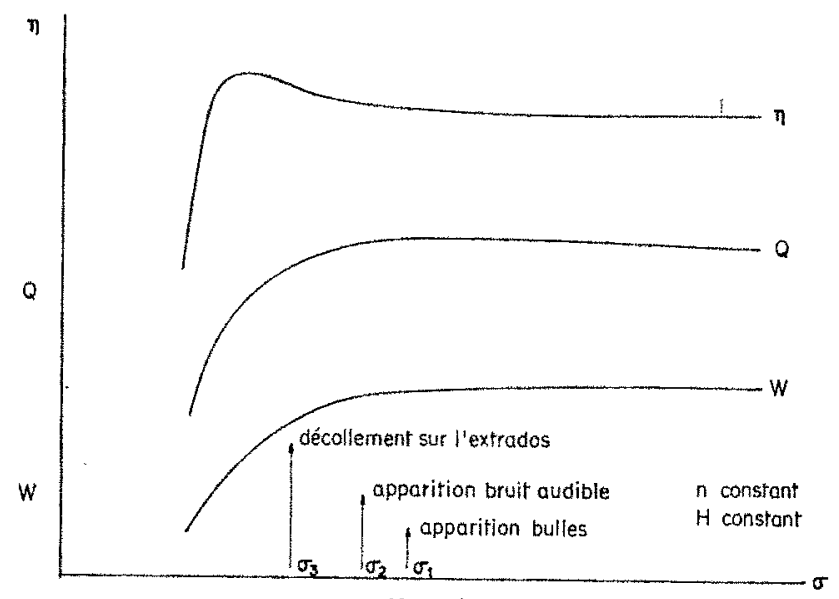

Fig. 4.

Courbes typiques de cavitation d'une turbine. Courbes typiques d'altération des paramètres pour un point donné.

représenté sur un diagramme $\sigma=f(Q)$ (fig. 5).

Il faut alors avoir un nombre de points suffisant pour que dans la colline générale de la turbine en débit-hauteur ou en puissance-vitesse on puisse tracer un résean de courbes équisigma.

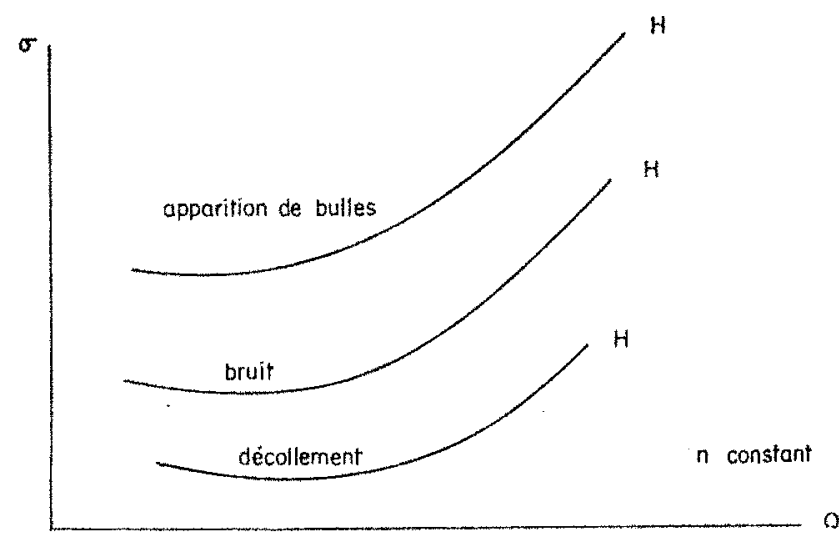

Frg. 5

Courbes typiques de cavitation d'une turbine. Ensemble des caractéristiques de cavitation de la machine.

Ce nombre de points peut varier de 10 à $\mathbf{3 0}$ selon la gamme d'utilisation de la turbine ou le type d'essai.

Le coefficient $\sigma$ est calculé en général pour une cote correspondant au plan des axes des aubes pour les turbines Kaplan, au plan médian du distributeur pour les autres machines à axe vertical, et sur l'axe de la roue pour les turbines à axe horizontal.

\section{PoMpes.}

Les courbes tracées habituellement représentent l'évolution de la hauteur, de la puissance et $\mathrm{du}$ rendement en fonction des conditions à l'entrée, c'est-à-dire du (NPSH), à vitesse de rotation et débit constants. Ces courbes correspondent à un point de fonctionnement donné de la pompe (fig. 6).

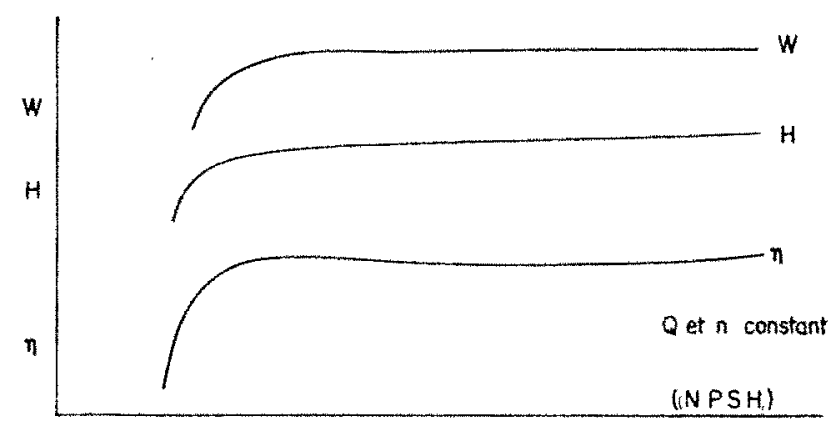

Fid. 6

Courbes typiques de cavitation des pompes. Alteration des paramètres caractéristiques pour un point de fonctionuement donné.

L'altération de la caractéristique peut être représentée par un diagramme $\mathrm{H}, \mathrm{W}$, $n$ en fonction du débit pour différentes conditions d'aspiration. Il existe également une autre représentation, se rapprochant de celle indiquée sur la figure 2 pour les hélices, qui consiste a caractériser l'apparition des diverses formes de cavitation pour une roue, ou plutôt un cillard donné, par des courbes (NPSH) $=f(Q)$ tracées pour chaque aspect de cavitation, par exemple

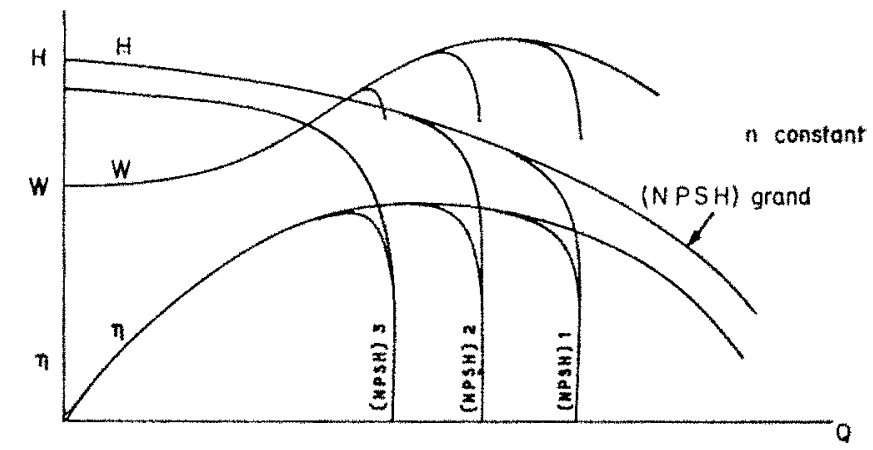

Fic. 7

Courhes typicues de cavitation des pompes. Alterration des caracteristiques globales. 


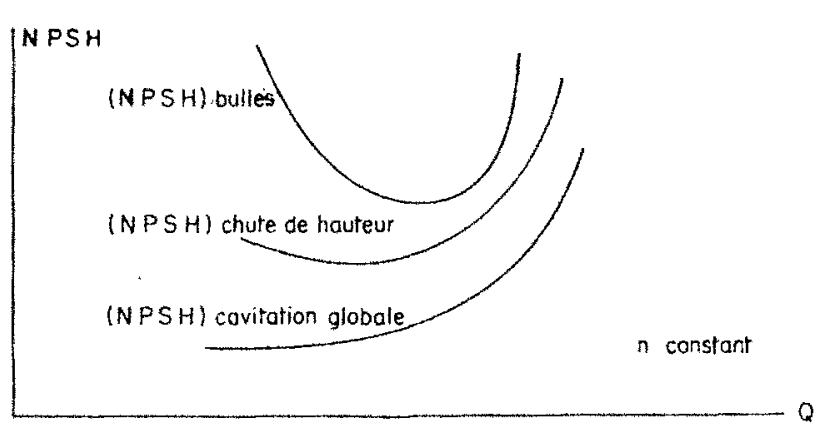

FiG. 8

Courbes typiques de cavitation des pompes. Caractéristiques globales de la pompe, rapportées à l'oillard.

apparition de bulles, cavitation industrielle (baisse de $\mathrm{H}$ de $3 \%$ ), cavitation globale, etc., (fig. 8).

Le NPSH est calculé en général pour l'altitude du centre de l'entrée de la roue, déterminé par l'intersection de l'axe de rotation de la machine avec le plan passant par l'extrémité amont des aubes.

$$
* *
$$

L'ensemble de ces courbes peut ainsi se classer en trois types différents :

- Les courbes établies pour un point de fonctionnement bien défini et montrant l'influence de la pression absolue sur les caractéristiques de ce point (fig. $3,4,6$ );

- Les courbes groupant un ensemble de résultats élémentaires dans un diagramme o (ou NPSH) en fonction d'une caractéristique de fonctionnement variable de la machine (fig. 2, 5. 8). De telles courbes peuvent être tracées pour chacune des manifestations de cavitation;

- Les courbes montrant l'altération de la caractéristique complète de la machine pour différentes conditions de pression absolue (fig. 3 , 7). Ce type de courbes ne pent se définir pour les turbines, par suite de l'existence d'un paramètre supplémentaire au moins : l'ouverture variable du distributeur.

\section{C-Détermination du seuil de cavitation cri- tique.}

En ce qui concerne les hélices, il n'est en général pas question de déterminer un seuil de cavitation critique; l'essai a généralement pour but de déterminer lapparition de la cavitation et, cette limite étant déteminée, les points de fonctionnement de l'hélice resteront en decà de celle-ci.
Ơn n'admet pas pour les pompes, de fonctionner en régime de cavitation, car les caractéristiques sont toujours rapidement perturbées par le fait que la cavitation se produit à l'entrée. Le seuil de cavitation industriel est basé sur une altération des performances, qui se produit toujours rapidement. Une chute de hauteur totale de $3 \%$ par rapport à sa valeur sans cavitation ponr le même débit et la même vitesse de rotation, est le critère généralement admis.

Les essais de turbines sur modèle réduit nécessitent par contre toujours la détermination d'un seuil de cavitation qui ne conduise pas à une cote d'implantation prohibitive, mais qui, d'autre part, n'entraine pas une altération trop marquée des performances. Ce seuil est appelé « sigma critique ; il est transposé (voir $\S \mathbf{E}$ ) de manière à conduire au sigma d'implantation.

La détermination du critique se fait soit par l'observation de l'altération des courbes de rendement, de débit ou de puissance, soit par la détermination visuelle et subjective d'un seuil de danger appelé $\sigma$ critique d’observation.

Dans la détermination du $\sigma$ critique d'après l'altération des courbes de rendement, certains caractérisent la valeur critique par le point où la courbe $\eta=f(\sigma)$ accuse une chute de rendement brusque et marquée; d'autres caractérisent la valeur critique par le point où le rendement a perdu $1 \%$ de sa valeur par rapport au rendement maximal obtenu avant la baisse de rendement ou par rapport à la valeur du rendement en régime non cavitant.

Lorsque la forme de la courbe de cavitation rend cette détermination difficile, notamment lorsqu'on se trouve dans une zone de fonctionnement oì le rendement diminue lentement depuis le début de l'abaissement de la pression absolue, certains se guident d'après la forme des courbes $\eta=f(\sigma)$ ainsi que $Q=f(\sigma)$ on $W=f(\sigma)$ Is déterminent ainsi trois valeurs critiques qui doivent être voisines. D'autres enfin prennent comme valeur critique la plus pessimiste des trois valeurs ainsi déterminées.

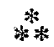

Cette revue des procédés montre bien qu'il est nécessaire en ce qui concerne les turbines d'établir une définition d'un sigma de référence présentant les avantages d'être:

- Significative: le critère utilisé doit correspondre à un état de cavitation déjà amorcé mais non dangereux;

- Fidèle : le seuil ainsi trouvé doit pouvoir 

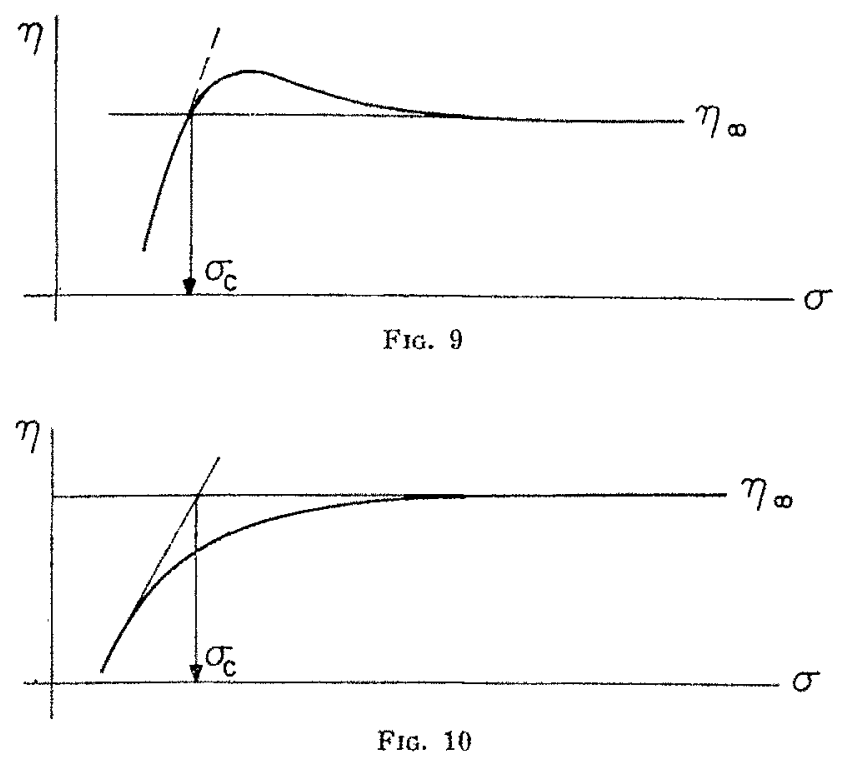

l'être avec fidélité quelle que soit la méthode utilisée d'un laboratoire à l'autre;

-.- Générale : il doit être possible d'utiliser la même définition pour tout point d'essai de la colline de fonctionmement, quelle que soit la forme de la courbe de cavitation relevée.

Assez fréquemment, on obtient des courbes ayant 1'aspect de la figure 9 :

Le rendement reste d'abord à pen près constant, présente un léger accroissement, puis s'effondre brusquement. C'est la figure qui apparait en principe sur les turbines Kaplan aux grandes ouvertures de roue, pour des angles d'attaque importants pour lesquels la cavitation apparaît assez brusquement sur l'ensemble de l'extrados de la pale.

Pour des ouvertures plus faibles, la cavitation s'établit progressivement sur des zones de plus en plus étendues, de sorte que le rendement présente une altération continue pouvant débuter pour des valeurs importantes de o (fig. 10).

Il peut enfin arriver que la courbe présente plusieurs paliers ou mềme quelques oscillations avant de s'effondrer (fig. 11).

Avant la cavitation développée, et notamment pour les cas compliqués comme celui de la figure 11 , il est fréquent que les points soient instables et non reproductibles d'un essai à l'autre. En fait, il n'y a plus, dans cette zone, de courbe à proprement parler, mais un nuage de points apparemment désordonnés. Dans ces conditions, il parait illusoire, pour ces formes de courbe, de définir un $\sigma$ critique en se donnant un écart par rapport au régime non cavitant ou au régime maximal. La valeur ainsi trouvée serait assez mal définie, même pour un laboratoire donné et a fortiori il n'y aurait que peu
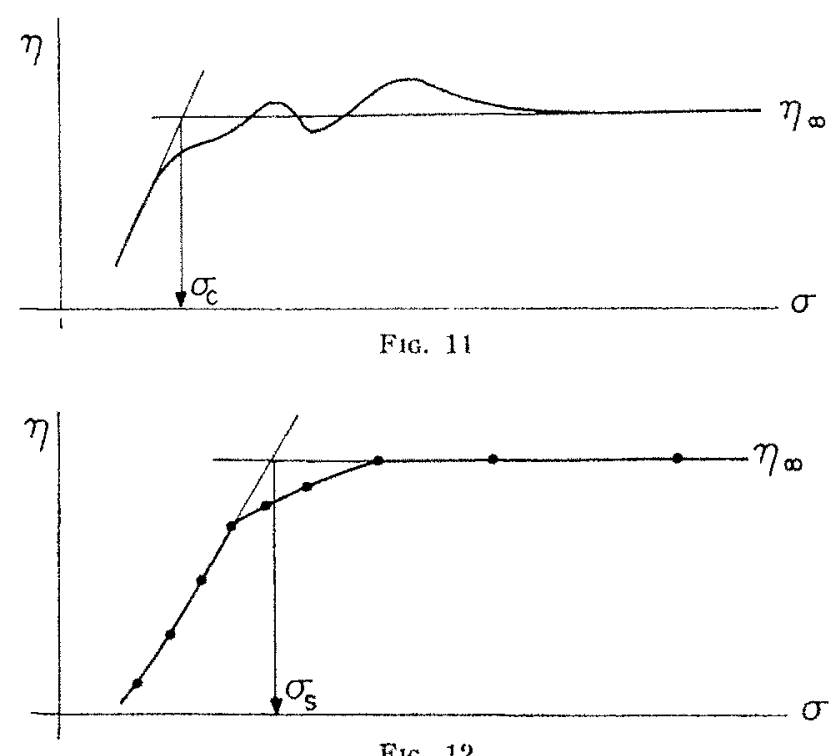

Fig. 12

d'espoir de trouver des valeurs comparables d'un laboratoire à un autre.

On constate en revanche que, lorsque la cavitation est franchement amorcée, les points de mesure se reproduisent avec plus de fidélité et sont sensiblement alignés sur une droite de forte pente descendante. C'est pourquoi nous proposons de prendre comme valeur d'un sigma que l'on pourrait appeler «sigma standard» l'abscisse de l'intersection de cette droite D avec la droite $\eta=\eta_{\infty}$ (fig. 12).

La valeur ainsi définie est bien déterminée et présente toute assurance de fidélité. C'est une valeur assez basse qui correspond en général à une cavitation déjả développée. II faut noter également que l'altération du rendement au point ainsi déterminé est parfois plus grande que celle qui serait admise dans un critère faisant référence à la valeur du rendement en régime non cavitant ou à cette valeur réduite de $1 \%$.

L'intérêt d'une telle méthode de détermination réside dans le fait que si le sigma critique est choisi avec une part importante d'appréciation personnelle, ce sigma standard devrait par contre permettre de comparer des machines differentes en utilisant une méthode conduisant, sans modificalions des installalions cxistantes dans les différents laboratoires, à des résultats pouvant facilement se comparer directement entre eux.

Le sigma d'installation donné par le constructeur serait alors présenté comme la somme du sigma standard et d'un $\Delta \sigma$ (positif ou négatif). Ce $\Delta \sigma$ représenterait la parl d'apprécialion personmelle du constructeur, résultant de son expérience dans le domaine en question, et déterminée par les méthodes traditionnelles qui sont propres à ses observations et à son installation. 


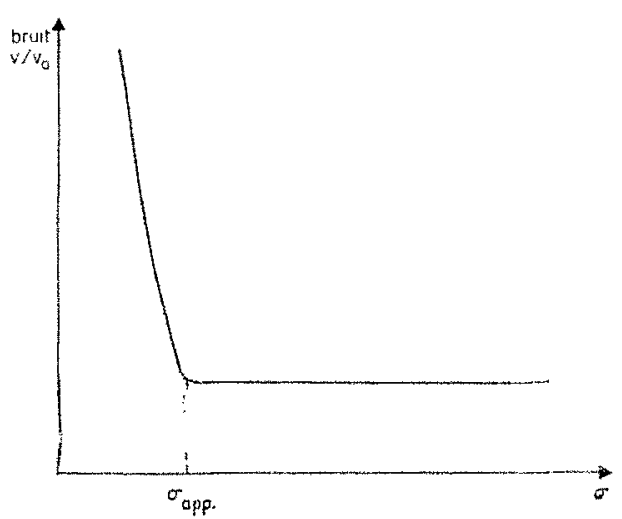

Fus. 13

\section{D-Détermination d'autres sigma particu- liers.}

Indépendamment de la détermination du $\sigma$ critique et du $\sigma$ standard, les observations faites au cours de l'essai permettent de déterminer des $\sigma$ d'apparition de diverses formes de cavitation, d'apparition de fluctuations de pression ou de bruit. Plusieurs laboratoires utilisent le critère du bruit pour la détermination de l'apparition de cavitation, avant même que les premières bulles soient nettement visibles. L'enregistrement du niveau global de bruit dans la zone en dépression d'une machine fournit une courbe selon la figure 13 sur laquelle un changement brusque de pente traduit l'apparition de cavitation avec une fidélité et une sensibilité excellentes, moyennant certaines précautions relatives à l'amortissement dû à la teneur en air de leau du circuit.

On peut également rechercher le spectre de fréquence du bruit ainsi relevé et étudier l'évolution d'une fréquence particulière. Il semble en effet que les bruits de haute fréquence soient parliculièrement influencés par: la présence de cavitation. L'analyse en amplitude du bruit relevé permet également de détecter l'apparition du phénomène et de suive son développement.

\section{E-Précision.}

La précision escomptée dans la détermination du $\sigma$ critique est variable, selon les installations et le type d'essais, de $\pm 2 \%$ à $\pm 10 \%$; il semble bien d'ailleurs que ces chiffres ne soient étayés que par la répétabilité de la courhe tracée, mais ne prennent pas en compte l'incertitude sur la détermination du $\sigma$ critique.

Une présentation globale de l'essai de cavitation d'une turbine par le tracé des collines de sigma présente l'avantage de donner une idée de la précision à escompter dans la détermination du o critique. En effet, les interpolations nécessaires à l'établissement d'une telle colline obligent à un lissage général des résultats, et la comparaison entre les valeurs $\sigma$ lissés et $\sigma$ mesurés fait apparaitre un écart qui peut être considéré comme significatif si le nombre de points d'essais est suffisant. L'exemple nous a été donné d'un essai comportant l'étude de vingt-cinq points de régime pour une turbine Kaplan, essai pour lequel l'écart quadratique moyen sur les valeurs les plus faibles de a atteignait $10 \%$ et $3 \%$ pour les valeurs les plus fortes.

\section{F-Marge de sécurité et détermination du sigma d'installation.}

L'ensemble des laboratoires interrogés estime que, au stade actuel des connaissances acquises soil par les utilisateurs, soit par les fournisseurs et leurs laboratoires, il n'est pas possible d'établir de règle objective définissant une marge de sécurité à donner, ou encore, ce qui revient au même, un effet d'échelle pour définir cette marge de sécurité.

Certains cependant recommandent la valeur 1,1 o critique comme valeur du $\sigma$ d'installation pour les machines Kaplan, par rapport à la valeur critique obtenue au moment de la chute des performances; d'autres utilisent plus empiriquement leur expérience, on la tenue des machines industrielles, pour définir leur marge de sécurité.

Fn ce qui concerne les turbines Francis, le problème semble encore plus mal défini : l'implantation serait trop optimiste en utilisant la marge de sécurité définie pour la machine Kaplan, et prohibitive en se guidant par l'apparition des torches; seule l'expérience de l'observateur semble être admise dans la détermination du $\sigma$ d'installation à partir du $\sigma$ critique d'observation.

Une telle technique subjective est évidemment sujette à critique, et il serait souhaitable que le jugement de l'observateur réglé dans un laboratoire déterminé et avec une méthode déterminée soit transformé en méthode fidèle et précise si les résultats en sont aussi satisfaisants.

En ce qui concerne les hélices marines, l'effet d'échelle le plus marqué semble être celui qui affecte les tourbillons d'extrémité : il arrive que l'eflet d'échelle sur le tourbillon soit tel, qu'une hélice ne présentant pas ce type de cavitation en modèle le présente sur le réel.

Signalons les essais effectués par le professeur Tenot (1934) sur une pompe-hélice fonctionnant à différentes vitesses et sous différents (NPSH), et qui l'ont conduit a la relation : 


$$
\frac{\sigma_{1}-\sigma_{c 1}}{\sigma_{2}-\sigma_{c 2}}=\frac{\mathrm{H}_{2}}{\mathrm{H}_{1}} \text { ou }\left(p_{1}-p_{c 1}\right)=\left(p_{2}-p_{c 2}\right)
$$

$\mathrm{H}_{1}, \sigma_{1}$ et $\sigma_{c 1}$ sont relatifs au modèle;

$\mathrm{H}_{2}, \tau_{2}$ et $\sigma_{62}$ sont relatifs au prototype;

ce qui signifie que le même écart absolu entre pression absolue sur le modèle et sur la machine industrielle est nécessaire pour que le même état de cavitation soit obtenu en des points homologues.

Il semble cependant à l'heure actuelle que ces essais, effectués sur une même roue de dimension faible par rapport à la pression absolue, ne puissent s'appliquer directement au cas de deux roues de dimensions très différentes ou au cas d'une roue de grande dimension verticale.

\section{CONCLUSION}

Nous avons présenté l'ensemble de ces méthodes d'essais en montrant leur diversité apparente et en essayant d'en tirer cependant les raisons qui conduisent à une telle diversité.

Les réponses à la dernière des questions que nous avions posées à chaque laboratoire et demandant l'avis de chacun concernant les modifications qu'il estimait nécessaire, montrent nettement le désir de chacun de pousser les recherches sur les moyens permettant de rendre les essais de cavitation au laboratoire plus efficaces et plus sûrs en ce qui concerne la prédiction du comportement de la machine industrielle.
A notre avis, un des premiers pas à faire dans cette voie est un eflort de systématisation qui permette à tous de parler un langage commun en ce qui concerne l'essai de cavitation luimême, son interprétation restant une affaire de jugement personnel à chaque opérateur.

Nous espérons avoir ainsi jeté les bases d'un texte qui pourrait conduire à une standardisation de la technique des essais de cavitation applicable, en général, dans les différents laboratoires sans modification majeure des pratiques actuelles.

\section{BIBLIOGRAPHIE}

[1] M. Fauconnet. - Le Laboratoire de Mécanique des fluides «Charmilles》 Bulletin Technique de la Sulsse Romande, 1961.

[2] Electricité de France. - Centre de Recherches et d'Essais de Chatou. «La Station d'essais de turbines 》, 1962.

[3] Osterwalder et LEGHER. - Le nouveau stand de cavitation de Escher Wyss. Bulletin Escher Wyss, $1956, \mathrm{n}^{\circ} 2$.

[4] Winternitz (F.A.L.). - The model turbine test facilities at the National Engineering Laboratory, East liilbride.

A.I.R.H. - Symposium de Nice —, 1960, (A-2).

[5] R. Dzialr.as. - Versuchsstände und Versuchseinrichtungen in den Versuchsanstalten Brünnenmühle und Hermaringen.

Voith Forschung und Konstruktion, 1958, Heft 4.

[6] S. Brnder. - La cavitation. Cours de dynamique du navire de l’Ecole Supérieure du Génie Militaire, 1960

[7] P. Danei - J. Duport. - Aspect of cavitation similitude in hydraulic turbines. A.I.R.Y. - $7^{\mathrm{e}}$ Congrès —, Lisbonne, 1957.

[8] R. T. KNAPP. - Gavitation scale effect. A.I.R.H. - 7e Congrès -, Lisbonne, 1957.
[9] H. Genber. - Some reflexions on modern scale formulas for cavitation phenomena. Symposium Teddington, 1955 (paper 11).

[10] P. Guiton. - Cavitation dans les pompes. S.H.F. - Commission de cavitation - Groupe de Irabail $n^{\circ} 2,1962$.

[11] A.-J. Stepanofr. - Pompes centrifuges et pompeshélices. (Chapitre XII). Dunod, 1961.

[12] J. Beaufrìre. - Description et définition des diverses figures de cavitation observées dans les machines et conduits hydrauliques. S.H.F. - Commission de cavitation - Groupe de travail n' 3 , 1962.

[13] J.-M. RATA. - Bruit de cavitation. A.I.R.H. - Symposium de Nice, 1960, (C-5).

[14] M. StrasienG. - Undissolved air cavities as cavitation nucles.

Symposium Teddington, 1955 (paper 6).

[15] J.-M. RatA. - La détermination acoustique du seuil de cavitation. Méthodes et appareillage utilisés. S.H.F. - «VIHes Journées de l'Hydrutlique », 1962, Question IV.

[16] P. Conmault. -- Incidence des teneurs en gaz de leau sur la mesure du bruit comme critère d'apparition de la cavitation. S.H.F. - «VIIes Jonrnées de l'Iydraulique », 1962, Question IV. 


\section{I S CUS I O N}

Président : M. Breqgeron

M. Ie Président remercie M. Crevalrer, qui a su présenter un rapport parfaitement objectif qui ne peut être contesté ni par les constructeurs, ni pay les utilisateurs. Un des points originaux du rapport est la défnition d’un o standard, sur lequel il serait nécessaire d'obtenir l'assentiment d'une majorité afin qu’il puísse éventuellement être utilisé contractuel lement.

La Commission ayant défini le a standard et non le o eritique, M. Cazenave demande à M. Chevaline ce cu'il faut contprendre lorsqu'il signale que les constructeurs prennent comne $\sigma$ industriel minimal la valeue $1,1 \sigma$ critique. A son sens, le $\sigma$ critique devrait précisément désigner la valeur minimale du a industriel.

M. Chrvalter répond que le $\sigma$ critique a été déterminé au Laboratoire par un procédé particulier à celui-ci et que, dan un cas bien défini, la marge de sécurité, prise entre le $y$ critique déterminé sur le modele et le o minimal d"implantation, est de $10 \%$.

A partir d'une défnition baséc sur le o standard, l'écart entre le $\sigma$ d'installation et le $\sigma$ standard se traduira par un $\Delta \sigma$ positif ou négatif propre a chaque laboratoire et traduisant le jugement de l'opérateur sur la transposition entre le $\sigma$ standard modele ef le $\sigma$ d'implantation.

M. Cazenaye demande s'il y aura deux collines de $\sigma$.

M. Chevalief répond qu'il y aura en effet la colline des a standards mesurée au laboratoire et la transposition de cette colline en $\sigma$ d'installation. La première est aisément contrôlable et comparahle entre deux muchines, alou's que la seconde ne pourra se contrôler que par des observations industrielles. C'est un processus qul est à rapprocher de la comparaison entre les collines de rendement sur modele et sur machine industrielle.
M1. le Président estime que ces considerations sortent du cadre de la réunion d'aujourd'hui, ce qui fait dire à M. Crisvalier que le souci de M. Cazbrave est de rattacher quelque chose que l'on connait bien à quelque chose que l'on a besoin de connaitre: le o d'implantation.

M. Comolex dit que la notion de critère, correspondant au $\sigma$ standard (on normalisể), paraît être d'un grand intérêt en vertu de sa dêfinition, qui est précise.

Cependant, comme on désigne habituellement sous le mème rocable o plusieurs rapports de nombres : coefficient de Thom: quotient (NPSH/H)..., il serait souhaitable de préciser lequel de ces rapports doit servix dans la définition de ce critere standard.

M. Casvaliter répond que, pour les turbines, e'est le $\sigma$ de Thoma utilisé dans I'essai de cavitation. Le (NPSH/HI) est particulier: aux pompes, mais, dans ce cas, il est peu probable gu*on soit amené à tuliliser une telle méthode pour la détermination $d u \sigma$ critique, car on s'arrête très tôt dans la cavitation des pompes. Chaque utilisateur definira sans doute son comme il le fait d'habitude.

M. Brnor féllcite M. Chevarifr et son groupe de travail pour l'excellente synthèse qu'ils ont réalisée en partant de points de vue et d'usages aussi différents que ceux qu'on rencontre dans l'étude des turbines, des hélices et des pompes. Il indique que, si le a standard a été détni à propos des turbines, il paraît parfaitement adapté au cas des hélices quand on s'intéresse à la chute des caractéristicues de fonctionnement; M. Bivon exprime done le souhatt que l'International Towing Tank Conference reprenne un jour à son compte ce standard.

M. le Président remercie M. Crevarrea de son travail, ainsi que les personnes ayant pris part à la discussion.

\section{NOTRE FRONTISPICE}

\section{(Cf. page 520)}

\section{GATVANT (1737-1798)}

De belles décotvertes scientificues ont parfois résulté d'un concours de circonstances sins rapport apparent avec leur objet. Co qu'on a appelé le galvanisme fut ainsi à l'origine de la pile. Louis Galvani, mérecin et physicien nè à Bologne en 1737, était depuis 1762 titulaire de la chaire d'anatonie à l'Université de Bologne, lorsqu'en 1786 il constata incidemment que des cuisses de grenoutlles écorchées, accrochées à un fil de cuivre suspendu à th balcoin de fer, étaient agitées de violentes convulsions lorsque le vent les amenait an contact du fer.

Galvani chercha sans grand succés la cause de cet étrange phénomène: it crut longtemos is l'infuence d'une electricit parti culiene, propre a l'economic "interne des grenouilles et suscite par le cerveau pour etre répandue dans le systcme nerveux. Mais lorsqunil se pencha à son tour sur la question, Volta, dexpérience en expérience, aboutit à la confection de la pile.

Le non de Galvant, dans les détivés qui ex ont été créés, est resté attaché aux contants continus enployés en théxapentique et en dectrolyse, à un appareil destiné à en mesurer l'intensité er à des procédés de recouvrenent d'un métal par un autre.

Lors de l'avènement de la République Cisalpine en 1797, Galvani prétéra renoncer à sa chaire que de prêter sernent à un ordre de choses qui heurtait ses convictions. Il mourut aे Bologine en 1798.

\section{GALVANI $(1737+7798)$}

Striking scientife discoweries sometimes result from apparently totally irrelevant combinations of circumstances.

It was in this wry that what ate now call galwanism led to tho invention of the electric battery.

Lingi Galwani, ioctor and natural philosopher, born in Balogna in 1737. had held the Chair of Anatomy at Bologna. Ytiversity since 1762 when, in 1786 he noted, qinte fortuitously, that skins cd frog's legs langing from an iron balcony by a copper zure "dcre "gnlvanised" into violent muscular activity when brought into contact with the iron by the wrind.

Galvani tried to find the cause of this odd phenomenon but whithout mich success. For a long time he enterfatied a theory of clectrical infuence proceding from the frog's owe internal ccosomy, in the form of thothses set ut by the brain and relayed throughout the nervous system. When $V$ olta in his titw, howeter, appled himself to the problem he usas led through a series of experiments, to the inyentwor of the electric battery. Galyonits name, by derivation, is applied to this day to such things as direct current entoloyed in physiotherapy and electrotysis, apparatus 1ised to measure the intensity of such current and the technique of sinc-plating whetals.

When the Cisalpine Republic was croated in 1707 Galyani chose to relinquish his Chair in preference to giving his allogionce to a system with which he was in profond disagreement.

He died in Bologna in 1798 . 